TRANSACTIONS OF THE

AMERICAN MATHEMATICAL SOCIETY

Volume 240, June 1978

\title{
THE SIGNATURE OF SYMPLECTIC MANIFOLDS
}

BY

LESLIE P. JONES

\begin{abstract}
The motivation for this work was a calculation by Oshanin of the image of the signature homomorphism from the special unitary cobordism ring into the integers. Here we compute this image for symplectic cobordism. This is accomplished by proving two divisibility theorems and then giving examples to show the theorems are the best possible.
\end{abstract}

I. Introduction. If $G$ equals any of the stable groups $O, S O, \operatorname{Spin}, U, S U$ or Sp we let $\Omega_{*}^{G}$ denote its associated cobordism ring, $B G$ its classifying space and $M G$ its Thom spectrum. For any oriented manifold $M$ we denote its signature by $\tau(M)$ (see e.g., Vick [23, p. 166]). For $G=S O, U, S U$ or Sp there is a homomorphism $\tau: \Omega_{*}^{G} \rightarrow Z$, the integers, defined by $\tau[M]=\tau(M)$.

Conner and Floyd [1] showed that Image $\left(\tau: \Omega_{4 k}^{S U} \rightarrow Z\right) \subseteq 2 Z$ for $k \neq 0$ (4) while Image $\left(\tau: \Omega_{16}^{S U} \rightarrow Z\right) \notin 2 Z$. Oshanin [13] proved a divisibility theorem and provided examples of $S U$-manifolds of minimal signature to show

$$
\begin{gathered}
\text { Image }\left(\tau: \Omega_{8 k+4}^{S U} \rightarrow Z\right)=16 Z, \\
\operatorname{Image}\left(\tau: \Omega_{16 k+8}^{S U} \rightarrow Z\right)=2 Z, \\
\operatorname{Image}\left(\tau: \Omega_{16 k}^{S U} \rightarrow Z\right)=Z .
\end{gathered}
$$

We will use a similar plan of attack to show

$$
\begin{gathered}
\text { Image }\left(\tau: \Omega_{8 k+4}^{\mathrm{Sp}} \rightarrow Z\right)=16 Z, \quad \operatorname{Image}\left(\tau: \Omega_{16 k+8}^{\mathrm{Sp}} \rightarrow Z\right)=4 Z, \\
\operatorname{Image}\left(\tau: \Omega_{32 k+16}^{\mathrm{Sp}} \rightarrow Z\right)=2 Z, \quad \operatorname{Image}\left(\tau: \Omega_{32 k}^{\mathrm{Sp}} \rightarrow Z\right)=Z .
\end{gathered}
$$

Nigel Ray [14] has computed $\Omega_{n}^{\text {Sp }}$ for $n<20$ and David Segal [18] has shown that there exists $[M] \in \Omega_{32}^{\mathrm{SP}}$ with $[M]=[\mathrm{RP}(2)]^{16}$ in $\Omega_{32}^{0}(\mathrm{RP}(2)$ is the real projective plane). Hence the top tangential Stiefel-Whitney number of $M$ is

$$
w_{32}(\tau)[M]=w_{32}(\tau)[\operatorname{RP}(2)]^{16}=w_{2}(\tau)[\operatorname{RP}(2)]=1
$$

(from [10, p. 53]) so $M$ has odd signature. Also, there is a forgetful homomorphism $\Omega_{*}^{\text {Sp }} \rightarrow \Omega_{*}^{S U}$ giving an upper bound on the size of Image $(\tau$ :

Received by the editors December 9, 1975 and, in revised form, September 7, 1976.

AMS (MOS) subject classifications (1970). Primary 55B20, 57D90.

Key words and phrases. Signature, cobordism, characteristic number, operation, Hopf algebra, multiplicative sequence. 
$\Omega_{*}^{\text {Sp }} \rightarrow Z$ ). Using the above we can form the following list (see $\S \mathrm{V}$ for details).

\begin{tabular}{c|c|c|c|c|c|c|c|c}
$4 k$ & 4 & 8 & 12 & 16 & 20 & 24 & 28 & 32 \\
\hline Image $\left(\tau: \Omega_{4 k}^{\text {Sp }} \rightarrow Z\right)$ & $16 Z$ & $4 Z$ & $16 Z$ & $2 Z$ & $16 Z$ or & $2 Z$ & $16 Z$ or & $Z$ \\
& & & & & $32 Z$ & $\begin{array}{c}4 Z \text { or } \\
8 Z\end{array}$ & $32 Z$ &
\end{tabular}

Note that since Image $\left(\tau: \Omega_{32}^{\mathrm{SP}} \rightarrow Z\right)=Z$ there is a containment relation

$$
\operatorname{Image}\left(\tau: \Omega_{4 k}^{S p} \rightarrow Z\right) \subseteq \operatorname{Image}\left(\tau: \Omega_{4 k+32}^{S p} \rightarrow Z\right)
$$

suggesting the possibility of periodic behavior like that in the $S U$ case. In what follows we will provide examples of symplectic manifolds in dimensions 20,24 and 28 with signatures 16,4 and 16 respectively. We will prove the following theorems.

THEOREM II. If $[M]$ is in $\Omega_{32 k+16}^{\mathrm{SP}}$, then $\tau[M]$ is even.

THEOREM III. If $[M]$ is in $\Omega_{16 k+8}^{\mathrm{SP}}$, then $\tau[M]=0$ (4).

Floyd [3] has defined a subalgebra $P \subset \Omega_{*}^{O}$. We prove

THEOREM I. If $[M]$ is in $P_{4 k+2}$, then $w_{4 k+2}(\tau)[M]=0$.

Floyd [3] proved that Image $\left(\Omega_{*}^{\mathrm{Sp}} \rightarrow \Omega_{*}^{9}\right) \subseteq P^{8}$ so that if $[M] \in \Omega_{32 k+16}^{\mathrm{Sp}}$ then $w_{32 k+16}(\tau)[M]=w_{32 k+16}(\tau)[N]^{8}=w_{4 k+2}(\tau)[N]$, some $N \in P_{4 k+2}$. This yields

$$
w_{32 k+16}(\tau)[M]=0
$$

so $M$ has even signature, establishing Theorem II. Theorem III also follows from Theorem I, but not so easily!

The background material for the proof of Theorem III is given in the next section. The proof is in $\S \mathrm{IV}$ while $\S \mathrm{V}$ gives the examples necessary to show that the theorems are the best possible. $\S \mathrm{VI}$ contains results on the signature of Spin manifolds.

The first five sections contain results of a thesis written under Professor Peter Landweber. §VI was also written under his guidance. His knowledge and encouragement were greatly appreciated. The author wishes to thank Professor Raphael Zahler for a series of informative meetings while the author and Professor Landweber were geographically separated. Finally, the author is indebted to the referee, Nigel Ray, for carefully reading the original version of this paper and making many suggestions about clarifying and condensing the exposition and to Ulrich Korshorke for his helpful conversations. Details of calculations may be found in the author's thesis.

II. Notation and background. Here we give notation and references used in $\S \S I I I$ and IV. We will use the following universal characteristic classes. 


$\begin{array}{ll}\text { Class } & \text { Name } \\ w_{i} \in H^{i}(B O ; Z / 2 Z) & \text { Stiefel-Whitney } \\ p_{i}^{S O} \in H^{4 i}\left(B S O ; Z\left[\frac{1}{2}\right]\right) & \text { Pontrjagin } \\ p_{i}^{\mathrm{Sp}} \in H^{4 i}(B \mathrm{Sp} ; Z) & \text { Symplectic Pontrjagin } \\ c_{i} \in H^{2 i}(B U ; Z) & \text { Chern }\end{array}$

For an exponent sequence $R=\left(n_{1}, \ldots, n_{k}\right)$ we define the degree of $R$ as $|R|=\sum j n_{j}$ and have characteristic classes

$$
\begin{gathered}
s^{R} \in H^{|R|}(B O ; Z / 2 Z) \cong(Z / 2 Z)\left[w_{1}, w_{2}, w_{3}, \ldots\right], \\
s_{S O}^{R} \in H^{4|R|}(B S O ; Z / 2 Z)=Z\left[\frac{1}{2}\right]\left[p_{1}^{S O}, p_{2}^{S O}, p_{3}^{S O}, \ldots\right], \\
{ }^{R} \in H^{4|R|}(B S \mathrm{Sp} Z Z) \cong Z\left[p_{1}^{\mathrm{Sp}}, p_{2}^{\mathrm{Sp}}, p_{3}^{\mathrm{Sp}}, \ldots\right] .
\end{gathered}
$$

These may be defined in terms of symmetrized monomials as described in Milnor [10, pp. 186-190], using the fact the cohomology groups mentioned are built up from symmetric functions. (For example, $w_{i}=s^{i \Delta_{1}}$ where $\Delta_{j}=$ $(0, \ldots, 0,1)$.) An exponent sequence $R=\left(n_{1}, \ldots, n_{k}\right)$ corresponds to a partition

$$
1, \ldots{ }_{n_{1}}, 1,2, \ldots \ddot{n}_{2}, 2, \ldots, k,{ }_{n_{k}}, k .
$$

Here we perpetuate the convention of Floyd [3] that an element of the cohomology of $B G$ is written with the same symbol as its Thom image in the cohomology of $M G$. The characteristic numbers he obtains are normal numbers and we will use $s^{R}[M], s_{S O}^{R}[M]$ and ${ }^{R} S^{R}[M]$ to mean normal numbers.

Floyd [3, p. 77], describes the Hopf algebra $A^{\prime}=$ the $(Z / 2 Z)$-vector space with basis $\left\{s^{R}\right\}$. We let $\delta, T, T^{+}, P_{k}$ and $P$ be as in Floyd [3, p. 78], so there are inclusions and identifications as follows

$$
S \subset T \subset A^{\prime} \cong H^{*}(B O ; Z / 2 Z) \text {. }
$$

We will write $\circ$ for the Hopf algebra product of Floyd [3]. For a given manifold we will denote its tangent and normal bundles by $\tau$ and $\nu$ respectively.

III. The proof of Theorem I. The original proof that $w_{4 k+2}$ vanishes tangentially on $P_{4 k+2}$ used the Landweber-Novikov operations (Landweber [9] and Novikov [11]) in unoriented cobordism and the Wu relations (Stong [19, p. 100] and Hsiang [6]). Floyd [3] asserts every element of $P_{4 k+2}$ is a Wall manifold (i.e., any Stiefel-Whitney number containing $w_{1}^{2}$ is 0 ). Landweber independently verified this result. Using $S q=1+S q^{1}+S q^{2}+\cdots$ and $v=1+v_{1}+v_{2}+\cdots$ for the total Steenrod square and Wu class we can show 


$$
S q^{2} w_{4 k}+v_{2} w_{4 k}=w_{4 k+2}+w_{1}^{2} w_{4 k}
$$

using the identity

$$
S q^{k}\left(w_{i}\right)=\sum_{0<t<k}\left(\begin{array}{c}
i-k+t-1 \\
t
\end{array}\right) w_{k-t} w_{i+\imath}, \quad k<i,
$$

from Hsiang [6]. The left-hand side is 0 tangentially by $\mathrm{Wu}$ (Stong [19, p. 100]) and the right-hand side reduces to $w_{4 k+2}$ on $P_{4 k+2}$. Thus $w_{4 k+2}(\tau)[M]=$ 0 . In fact, Stong informed the author that $w_{4 k+2}=\left(v_{2 k+1}\right)^{2}$ and $v_{2 k+1}$ is divisible by $w_{1}$.

IV. The proof of Theorem III. Here we show that Image $\left(\tau: \Omega_{16 k+8}^{\mathrm{Sp}} \rightarrow Z\right) \subseteq$ 4Z. Let $\Re^{\prime}=\left\{R \mid R=\left(0, n_{2}, 0, n_{6}, 0, n_{14}, 0, \ldots, 0, n_{2^{\prime}-2}\right)\right\}$ and let $\Re_{n}^{\prime}$ be the set of all elements of $\Re^{\prime}$ of degree $n$. We then prove Theorem III by proving the following lemmas.

LEMma A. If $[M] \in \Omega_{16 k+8}^{\mathrm{Sp}}$, then $\tau[M]=\Sigma_{R \in \mathfrak{R}^{\prime}} S^{R}[M] \bmod 4$.

LeMma B. $\Sigma_{R \in \mathscr{R}}, s^{R}$ vanishes on $P_{4 k+2}$. In fact, $w_{2 n}(\tau)[M]=$ $\Sigma_{R \in \mathscr{R}_{2 n}^{\prime}} s^{R}[M]$, any $[M] \in \Omega_{2 n}^{O}$.

LEMMA $C$. Let $R$ be some set of exponent sequences which all have the same degree $4 k+2$. If $\Sigma_{R \in \mathscr{R}} s^{R}$ vanishes on $P_{4 k+2}$, then $\Sigma_{R \in \mathscr{R}}{ }^{\prime} S^{R}[M]=0$ (4), any $[M] \in \Omega_{16 k+8}^{\mathrm{Sp}}$.

The reader is referred to Hirzebruch [5, Chapter 1], for the definition and important properties of multiplicative sequences.

Proof of Lemma A. Using the results of Floyd and Segal will require writing the signature mod 4 of $[M] \in \Omega_{16 k+8}^{\mathrm{Sp}}$ in terms of the normal numbers $' S^{R}[M]$. The signature is often computed using the $L$-polynomials of the Hirzebruch signature theorem. We write $L_{k}^{S O}(\tau)$ for the $k$ th polynomial. They are in the form

$$
\frac{1}{\text { odd }} \text { (an integral polynomial in tangential Pontrjagin classes) }
$$

and may be thought of as polynomails with coefficients in $Z_{(2)}=$ integers localized at $2([5, \mathrm{p} .13])$. Changing to normal Pontrjagin numbers does not change this last fact. From the relationship $\left(p^{\mathrm{Sp}}\right)^{2}=p^{\text {so }}$ of Stong [20, p. 426], it follows that the signature of a $4 k$-dimensional symplectic manifold can be written as a linear combination over $Z_{(2)}$ of the characteristic numbers $' S^{R}[M]$. This polynomial will be denoted $L_{k}^{\mathrm{Sp}}(\nu)$. The polynomial is in the form

$$
L_{k}^{\mathrm{Sp}}(\nu)[M]=\frac{1}{\operatorname{odd}}\left(\sum_{|R|=k} m_{R} S^{R}[M]\right), \quad m_{R} \in Z .
$$

In the case of a $16 k+8$-dimension symplectic manifold the numbers 
${ }^{\prime} S^{R}[M]$ are all even (Segal [18]) so any ${ }^{R}{ }^{R}[M]$ with an even coefficient will contribute a multiple of 4 . Hence the $m_{R}$ 's which are odd must be precisely found. In particular, we need an explicit expression for the polynomial $L_{k}^{\mathrm{Sp}}(\nu)_{\bmod 2}$, that is, with the coefficients reduced mod 2. For an example,

$$
L_{2}^{S O}(\tau)=\frac{1}{45}\left(7 p_{2}^{S O}(\tau)\right)-\left(p_{1}^{S O}(\tau)\right)^{2}
$$

while

$$
L_{2}^{\mathrm{Sp}}(\nu)=\frac{1}{45}\left(17^{\prime} S^{\Delta_{2}}+20^{\prime} S^{2 \Delta_{1}}\right)
$$

so that $L_{2}^{\mathrm{Sp}}(\nu)_{\bmod 2}={ }^{\Delta_{2}}$.

To obtain an explicit expression for $L_{k}^{\mathrm{Sp}}(\nu)_{\bmod 2}$ we turn to the series

$$
Q(z)=1+\sum_{k=1}^{\infty}(-1)^{k-1} \frac{2^{2 k}}{(2 k) !} B_{k} z^{k}
$$

which generates the $L$-polynomials $\left(B_{k}=k\right.$ th Bernoulli number). For a rational number $r$ with $r=2^{e_{2}} \cdot 3^{e_{3}} \cdot 5^{e_{5}} . \ldots$ let $\nu_{p}(r)=e_{p}$.

Sublemma.

$$
\nu_{2}\left(\frac{2^{2 k}}{(2 k) !} B_{k}\right) \begin{cases}=0, & k=2^{l}, \\ >0, & \text { otherwise. }\end{cases}
$$

Proof. From Milnor [10, p. 284], the denominator of $B_{k}$ (in lowest terms) is square free and divisible by 6 so that $\nu_{2}\left(B_{k}\right)=-1$. For a prime $p$,

$$
\nu_{p}(k !)=\left[\frac{k}{p}\right]+\left[\frac{k}{p^{2}}\right]+\left[\frac{k}{p^{3}}\right]+\cdots \quad([4, \mathrm{p} .342])
$$

where $[x]=$ the greatest integer in $x$. Then $\nu_{2}\left(\left(2^{l}\right) !\right)=\left[2^{l} / 2\right]+\left[2^{l} / 4\right]$ $+\cdots+\left[2^{l} / 2^{l}\right]=2^{l-1}+2^{l-2}+\cdots+1=2^{l}-1$.

Since this gives the largest possible value we have

$$
\nu_{2}(k !) \begin{cases}=k-1, & k=2^{l}, \\ <k-1, & k \neq 2^{l} .\end{cases}
$$

Then

$$
\nu_{2}\left(\frac{2^{2 k}}{(2 k) !} B_{n}\right)=2 k-1-v_{2}((2 k) !) \begin{cases}=0, & k=2^{l}, \\ >0, & k \neq 2^{l} .\end{cases}
$$

This shows the natural map $Z_{(2)}[[z]] \rightarrow(Z / 2 Z)[[z]]$ sends $Q(z)$ to the series

$$
Q(z)_{\bmod 2}=1+z+z^{2}+z^{4}+\cdots+z^{2^{k}}+\cdots .
$$

For $\tau$ and $\nu$ the tangent and normal bundles of some symplectic manifold we have

$$
p^{S o}(\tau)=\left(p^{\mathrm{SP}}(\tau)\right)^{2}=\left(p^{\mathrm{Sp}}(\nu)^{-1}\right)^{2}=\left(p^{\mathrm{Sp}}(\nu)\right)^{-2}
$$


so $L^{\mathrm{Sp}}(\nu)_{\bmod 2}$ is the multiplicative sequence generated by the series

$$
\begin{aligned}
& \left(1+z+z^{2}+z^{4}+z^{8}+\cdots+z^{2^{k}}+\cdots\right)^{-2} \\
& \quad=1+z^{2}+z^{6}+z^{14}+\cdots+z^{2^{k}-2}+\cdots=1+\sum z^{2^{k}-2} .
\end{aligned}
$$

If we have a power series $R(z)=1+b_{i} z^{i}$ over $Z / 2 Z$ then the coefficient of ' $S^{R}$ in the resulting multiplicative sequence is $b_{1}^{n_{1}} b_{2}^{n_{2}} \cdots \cdots b_{k}^{n_{k}}$ where $R=$ $\left(n_{1}, n_{2}, \ldots, n_{k}\right)$. Here we have $R(z)=1+\sum z^{2^{k}-2}$ so the coefficient of ' $S^{R}$ is 1 if and only if $R \in \Re^{\prime}$. Hence

$$
L^{\mathrm{Sp}}(\nu)_{\bmod 2}=1+\sum_{R \in \mathscr{R}^{\prime}} S^{R}
$$

and $\tau[M]$ for $[M] \in \Omega_{16 k+8}^{\mathrm{Sp}}$ is determined $\bmod 4$ by $\Sigma_{R \in \mathscr{R}^{\prime}} S^{R}[M]$.

For example,

$$
L^{\mathrm{Sp}}(\nu)_{\bmod 2}=1+\underbrace{1+S^{\Delta_{2}}}_{L_{2}^{S p}(\nu)_{\bmod 2}}+\underbrace{\prime S^{2 \Delta_{2}}}_{L_{4}^{S p}(\nu)_{\bmod 2}}+\underbrace{S^{\Delta_{6}}+' S^{3 \Delta_{2}}}_{L_{b}^{S p}(\nu)_{\bmod 2}}+\cdots
$$

Proof of Lemma B. We will use the following fact.

Sublemma. If $[M] \in \Omega_{n}^{O}$, then $s^{2 R}[M]^{2}=s^{R}[M], s^{R^{\prime}}[M]^{2}=0$ if $R^{\prime} \neq 2 R$.

Proof. From Switzer [22, p. 383], we have

$$
S^{R}[M \times N]=\sum_{R_{1}^{\prime}+R_{2}^{\prime}=R^{\prime}} s^{R_{1}^{\prime}}[M] \cdot s^{R_{2}^{\prime}}[N]
$$

so that

$$
s^{R^{\prime}}[M]^{2}=\sum_{R_{1}^{\prime}+R_{2}^{\prime}=R^{\prime}} s^{R_{1}^{\prime}}[M] \cdot s^{R_{2}^{\prime}}[M] .
$$

Then the only terms which do not cancel mod 2 are those with $R_{1}^{\prime}=R_{2}^{\prime}$.

Let $L^{S O}(\nu)$ be the multiplicative sequence derived from $(Q(z))^{-1}$. It gives $\tau[M]$ in terms of $s_{S O}^{R}[M]$ 's. Also, let $L^{S O}(\nu)_{\bmod 2}$ be derived from

$$
\left(Q(z)_{\bmod 2}\right)^{-1}=1+z+z^{3}+z^{7}+\cdots+z^{2^{k}-1}+\cdots .
$$

We see that $L^{S O}(\nu)_{\bmod 2}=1+\Sigma_{R \in \Re_{R}^{\prime \prime}} S_{S O}^{R}[M]$ where

$$
\Re^{\prime \prime}=\left\{R \mid R=\left(n_{1}, 0, n_{3}, 0, \ldots, 0, n_{7}, 0, \ldots, n_{2^{k}-1}, 0, \ldots\right)\right\} .
$$

Applying the cohomology mod 2 reduction map

$$
\rho_{2}: H^{*}(-; Z) \rightarrow H^{*}(-; Z / 2 Z)
$$

we can deduce

$$
\rho_{2}\left(s_{s \delta}^{\left(n_{1}, n_{2}, \ldots, n_{k}\right)}\right)=s^{\left(0,2 n_{1}, 0,2 n_{2}, 0 \cdots 0,2 n_{k}\right)}
$$

from the fact $\rho_{2}\left(p_{n}^{s O}\right)=\left(w_{2 n}\right)^{2}$. Then $s^{R}$ will appear in $\rho_{2} L^{S O}(\tau)$ iff $R=$ $\left(0, n_{2}, 0, \ldots, 0, n_{6}, 0, \ldots, 0, n_{2^{k}-2}, 0, \ldots\right)$ with each entry even. Hence 


$$
\rho_{2} L^{S O}(\nu)=1+\sum_{R \in \Re^{\prime}} s^{2 R}
$$

For closed manifold $M$ there exists an oriented manifold $N$ with [N] = $[M]^{2}$ in $\Omega_{*}^{Q}$. If $\operatorname{dim} M=2 n$, then $\operatorname{dim} N=4 n$ and we have

$$
\begin{aligned}
L_{n}^{S O}(\tau)[N] & =\tau[N] \equiv w_{4 n}(\tau)[N] \bmod 2 \\
& =w_{4 n}(\tau)[M]^{2}=w_{2 n}(\tau)[M]
\end{aligned}
$$

so $\rho_{2} L_{n}^{S O}(\tau)[N]=w_{2 n}(\tau)[M]$. Recall that $L_{n}^{S O}(\tau)[N]=L_{n}^{S O}(\nu)[N]$ and the polynomials are the same elements of $H^{*}(N, Z)$. Combining the above facts we have

$$
\begin{aligned}
w_{2 n}(\tau)[M] & =\rho_{2} L_{n}^{S O}(\tau)[N]=\rho_{2} L_{n}^{S O}(\nu)[N]=\sum_{R \in \mathscr{R}_{2 n}^{\prime}} s^{2 R}[N] \\
& =\sum_{R \in \mathscr{R}_{2 n}^{\prime}} s^{2 R}[M]^{2}=\sum_{R \in \mathscr{R}_{2 n}^{\prime}} s^{R}[M], \text { as claimed. }
\end{aligned}
$$

If $2 n=4 k+2$ and $[M] \in P_{4 k+2}$ we must have $\Sigma_{R \in R^{\prime}{ }_{4 k+2}} s^{R}[M]=0$ since $w_{4 k+2}(\tau)[M]=0$ by Theorem I.

Proof of Lemma C. Since ' $S^{R}[M]=0$ (2) for $[M] \in \Omega_{16 k+8}^{\mathrm{Sp}},|R|=4 k+$ 2, we may define a pairing

$$
A_{4 k+2}^{\prime} \otimes \Omega_{16 k+8}^{\mathrm{Sp}} \rightarrow Z / 2 Z
$$

by $s^{R} \otimes[M] \mapsto\left(\frac{1}{2} ' S^{R}[M]\right)_{\bmod 2}$. Using Floyd [3], we can prove that

$$
' S^{\Delta_{2}} \circ{ }^{R} S^{R}[M]=0(4), \quad[M] \in \Omega_{8 k}^{\mathrm{Sp}}, \quad|R|=2 k-2,
$$

as a corollary of his (3.5), p. 85, using the same proof as (3.6). Floyd himself shows

$$
{ }^{\prime} S^{n \Delta_{1}} \circ{ }^{\prime} S^{R}[M]=0(4), \quad[M] \in \Omega_{8 k}^{\mathrm{Sp}}, \quad|R|=2 k-n .
$$

Since $16 k+8=8(2 k+1)$ we conclude that $T^{+} \circ A^{\prime}$ vanishes under this pairing so we really have a pairing

$$
\left(A^{\prime} / T^{+} \circ A^{\prime}\right)_{4 k+2} \otimes \Omega_{16 k+8}^{\mathrm{Sp}} \rightarrow Z / 2 Z \text {. }
$$

But

$$
\left(A^{\prime} / T^{+} \circ A^{\prime}\right)_{n} \otimes P_{n} \rightarrow Z / 2 Z
$$

is a dual pairing. Thus if $\Sigma_{R \in \mathfrak{R}} s^{R}$ vanishes under (**) it vanishes under (*) also so that $\sum_{R \in \Re} \frac{1}{2}{ }^{\prime} S^{R}[M]=0$ (2) or $\Sigma_{R \in \Re}{ }^{\prime} S^{R}[M]=0$ (4), as claimed.

Combining these results we conclude

$$
\operatorname{Image}\left(\tau: \Omega_{16 k+8}^{\mathrm{Sp}} \rightarrow Z\right) \subseteq 4 Z \text {. }
$$

V. Construction of examples. In this section we demonstrate the existence of symplectic manifolds in dimensions 20,24 and 28 with signatures 16,4 and 16 
respectively. Generators given by Ray [14] are listed which provide the necessary examples in lower dimensions.

Stong [20] gives a method for constructing numerous symplectic manifolds. Let $N=C P\left(2 k_{1}-1\right) \times \cdots \times C P\left(2 k_{s}-1\right), s$ even, and $\xi_{i}$ be the canonical bundle over $C P\left(2 k_{i}-1\right)$ pulled back over $N$. If $a_{i} \in c_{1}\left(\xi_{i}\right)$ and $a=a_{1}$ $+\cdots+a_{s} \in H^{2}(N ; \mathrm{Z})$ then the symplectic manifold dual to $\left(-a^{2}\right)^{j}$ will be denoted here by $n_{j}\left(k_{1}, \ldots, k_{s}\right)$. Note that

$$
\operatorname{dim} n_{j}\left(k_{1}, \ldots, k_{s}\right)=4\left(k_{1}+\cdots+k_{s}\right)-2 s-4 j \text {. }
$$

The symplectic Pontrjagin numbers of such a manifold can be mechanically computed.

Even these manifolds, however, are not adequate for providing all the generators for $\Omega_{4 k}^{\mathrm{Sp}}$ or even finding manifolds of minimal signature. We must then try to divide existing cobordism classes by integers. We use $K O$-theory (real $K$-theory) to see when this may be done. There is a generalized Hurewicz map (Ray [15, p. 284]) called the Sp Hattori-Stong map

$$
\Omega_{*}^{\mathrm{Sp}} \stackrel{k o}{\rightarrow} K O_{*}(M \mathrm{SP}), \quad[15, \mathrm{p} .293]
$$

and we use the notation of Ray [15, pp. 284 and 288], for $K O_{*}(M \mathrm{Sp})$. Kochman [8] and Okita [12] show that $k o$ is split monic in dimensions $<30$. Hence, in these dimensions if $k o[M]$ is divisible by an integer $n$ then $[M] / n$ exists in $\Omega_{*}^{\mathrm{Sp}}$. In particular, if $k o[M]=0(2),[M] / 2$ exists in $\Omega_{*}^{\mathrm{Sp}}$.

Ray [14] lists generators for $\Omega_{4 k}^{\mathrm{SP}}, k=1,2,3,4$, and we list the ones we need in this notation below.

$$
\begin{array}{llll}
\operatorname{dim} & \multicolumn{1}{c}{[M]} & k o[M]_{\bmod 2} & \tau[M] \\
4 & 2 x_{1}=n_{1}(1,1,1,1) & x & -16 \\
8 & 2 x_{2}=n_{1}(2,2) & x \sigma_{1}^{\prime} & 4 \\
12 & x_{1} x_{2} & y \sigma_{1}^{\prime}+x \sigma_{2}^{\prime} & -16 \\
& 2 x_{3}=n_{1}(2,3) & x\left(\sigma_{1}^{\prime}\right)^{2} & 0 \\
16 & \frac{1}{2}\left(x_{2}^{2}+x_{1} x_{3}\right) & y\left(\sigma_{1}^{\prime}\right)^{2}+x \sigma_{3}^{\prime} & 2
\end{array}
$$

Further calculations (in the author's thesis) have shown that

$$
k o\left(\frac{1}{16} n_{2}(2,6)\right)_{\bmod 2}=x\left(\sigma_{1}^{\prime}\right)^{4}+x \sigma_{1}^{\prime} \sigma_{3}^{\prime}
$$

and that $\tau\left(\frac{1}{16} n_{2}(2,6)\right)=0$. Also, $\operatorname{dim} n_{2}(2,6)=20$.

In dimension 20 ,

$$
\left(2 x_{1}\right)\left(\frac{1}{2}\left(x_{2}^{2}+x_{1} x_{3}\right)\right)+\left(x_{1}^{2}\right)\left(2 x_{3}\right)=x_{1} x_{2}^{2}+3 x_{1}^{2} x_{3}
$$

has signature -32 and $k o$-image even, so Image $\left(\tau: \Omega_{20}^{\mathrm{Sp}} \rightarrow Z\right)=16 Z$. In dimension 24 , the manifold 


$$
\left(2 x_{2}\right)\left(\frac{1}{2}\left(x_{2}^{2}+x_{1} x_{3}\right)\right)+\left(2 x_{3}\right)\left(x_{1} x_{2}\right)=x_{2}^{3}+3 x_{1} x_{2} x_{3}
$$

has signature 8 and $k o$-image even, so Image $\left(\tau: \Omega_{24}^{\mathrm{Sp}} \rightarrow Z\right)=4 Z$.

Direct computation shows no example of an $[M] \in \Omega_{28}^{\mathrm{Sp}}$ can be constructed out of classes from Ray [14]. We do, however, have the manifold

$$
\left(x_{1} x_{2}\right)\left(\frac{1}{2}\left(x_{2}^{2}+x_{1} x_{3}\right)\right)+\left(x_{1} x_{2}\right)\left(x_{2}^{2}\right)+\left(2 x_{3}\right)\left(x_{2}^{2}\right)+\left(x_{1}^{2}\right)\left(\frac{1}{16} n_{2}(2,6)\right)
$$

which has signature -96 and $k o$-image even so $\operatorname{Image}\left(\tau: \Omega_{28}^{\mathrm{Sp}} \rightarrow Z\right)=$ $16 Z$. Thus we have shown that Theorems II and III are the best possible.

VI. The spin case. We can compute Image $\left(\tau: \Omega_{4 n}^{\text {Spin }} \rightarrow Z\right)$ quite easily using results from the literature. Rohlin [16] has shown that Image $\left(\tau: \Omega_{4}^{\text {Spin }} \rightarrow Z\right)=$ 16Z. Also, the quaterionic projective plan $H P(2)$ is a Spin manifold and its signature is 1 so that $\operatorname{Image}\left(\tau: \Omega_{8}^{\text {Spin }} \rightarrow Z\right)=Z$. Finally, Stong [21] proved that the forgetful homomorphism

$$
\Omega_{8 k+4}^{S U} \rightarrow \Omega_{8 k+4}^{\text {Spin }} / \text { Torsion }
$$

is epic. From Oshanin's results on the signature of $S U$ manifolds we then have the following list.

$$
\operatorname{Image}\left(\tau: \Omega_{8 k+4}^{\text {Spin }} \rightarrow Z\right)=16 Z, \quad \operatorname{Image}\left(\tau: \Omega_{8 k}^{\text {Spin }} \rightarrow Z\right)=Z .
$$

\section{BIBLIOGRAPHY}

1. P. E. Conner and E. E. Floyd, Torsion in SU-bordism, Mem. Amer. Math. Soc., No. 60 (1966). MR 32 \#6471.

2. The relation of cobordism to K-theories, Lecture Notes in Math., vol. 28, SpringerVerlag, Berlin and New York, 1966. MR 35 \#7344.

3. E. E. Floyd, Stiefel-Whitney numbers of quaternionic and related manifolds, Trans. Amer. Math. Soc. 155 (1971), 77-94. MR 42 \#8509.

4. G. H. Hardy and E. M. Wright, An introduction to the theory of numbers, Oxford Univ. Press, Cambridge, 1960.

5. F. Hirzebruch, Topological methods in algebraic geometry, 3rd ed., Springer-Verlag, New York, 1966. MR 34 \#2573.

6. Wu-Chung Hsiang, On Wu's formula of Steenrod squares on Stiefel-Whitney classes, Bol. Soc. Mat. Mexicana (2) 8 (1963), 20-25. MR 29 \#609.

7. Leslie P. Jones, On the image of the signature homomorphism for symplectic cobordism, Doctoral Thesis, Rutgers Univ., August, 1975.

8. Stanley Kochman, Personal communication to Peter S. Landweber, dated August 11, 1974.

9. P. S. Landweber, Cobordism operations and Hopf algebras, Trans. Amer. Math. Soc. 129 (1967), 94-110. MR 36 \#2145. 1974.

11. S. P. Novikov, Methods of algebraic topology from the viewpoint of cobordism theory, Izv. Akad. Nauk SSSR Ser. Mat. 31 (1967), 855-951 = Math. USSR Izv. 1 (1967), 827-922. MR 36 \#4561.

12. Rikio Okita, On the MSp Hattori-Stong problem, Osaka J. Math. 13 (1976).

13. S. D. Ošanin, The signature of SU-manifolds, Mat. Zametki 13 (1973), 97-102 = Math. Notes 13 (1973), 57-61. MR 49 \#3999. 
14. Nigel Ray, Realizing symplectic bordism classes, Proc. Cambridge Philos. Soc. 71 (1972), 301-305.

15. Some results in generalized homology, K-theory and bordism, Proc. Cambridge Philos. Soc. 71 (1972), 283-300.

16. V. A Rohlin, New results in the theory of four-dimensional manifolds, Dokl. Akad. Nauk SSSR (N.S.) 84 (1952), 221-224. (Russian) MR 14, 573.

17. David Segal, Symplectic cobordism of manifolds, M. I. T., Cambridge Mass., 1968; On the symplectic cobordism ring, Comment. Math. Helv. 45 (1970), 159-169. MR 41 \#7699.

18. __ Divisibility conditions on characteristic numbers of stably symplectic manifolds, Proc. Amer. Math. Soc. 27 (1971), 411-415. MR 42 \#5282.

19. Robert E. Stong, Notes on cobordism theory, Princeton Univ. Press, Princeton, N. J.; Univ. of Tokyo Press, Tokyo, 1968. MR 40 \#2108.

20. Some remarks on symplectic cobordism, Ann. of Math. (2) 86 (1967), 425-433. MR 36 \#2162.

21. —_ Relations among characteristic numbers. II, Topology 5 (1966), 133-148. MR 33 \#741.

22. Robert M. Switzer, Algebraic topology-homotopy and homology, Springer-Verlag, New York, 1975. MR 52 \#6695.

23. James W. Vick, Homology theory, Academic Press, New York, 1973. MR 51 \# 11475.

Department of Mathematics, Rutgers University, New Brunswick, New Jersey 08903

Current address: Department of Computer Science, Pennsylvania State University, University Park, Pennsylvania 16802 